\title{
A new method for functional analysis of plastid EMBRYO-DEFECTIVE PPR genes by efficiently constructing cosuppression lines in Arabidopsis
}

\author{
Jingli Chen, Haojie Zhu, Jirong Huang and Weihua Huang* ${ }^{*}$
}

\begin{abstract}
Background: Pentatricopeptide-repeat proteins (PPRs) characterized by tandem arrays of a degenerate 35-aminoacid repeat (PPR motif) can bind a single strand RNA and regulate organelle gene expression at the post-transcriptional level, including RNA cleavage, splicing, editing and stability etc. PPRs are conserved in all eukaryotes and extremely expanded in higher plants. Many knockout mutants of PPR genes are embryonically lethal. These genes are named EMBPPRs and functional analysis of them is hindered by the difficulty in obtaining their knockout mutants.

Results: Here, we report a new method for functional analysis of plastid EMB PPRs by efficiently constructing their cosuppression lines in Arabidopsis. When we overexpressed a mutated full length or truncated coding sequence (CDS) of EMB PPRS, such as EMB2279, EMB2654 and EMB976 (all belong to the P family PPRs) in the wild-type (WT) background, a large portion of $\mathrm{T}_{1}$ plants displayed chlorosis phenotypes, which are similar to those of the weak allele mutants, knockdown lines or partially complementary lines. RT-PCR analysis showed that overexpression of the truncated EMBPPRs led to significant and specific downregulation of their corresponding endogenous mRNAs. However, when these EMB PPRs were overexpressed in the Post transcriptional Gene Silencing (PTGS) deficient mutant, RNAdependent RNA polymerase 6 ( $r d r 6)$, none of the $T_{1}$ plants displayed chlorosis phenotypes. These results indicate that the chlorosis phenotype results from post transcriptional silencing of the corresponding endogenous gene (also known as sense cosuppression).
\end{abstract}

Conclusions: Overexpression of an appropriately truncated EMB PPR CDS in WT leads to gene silencing in a RDR6dependent manner, and this method can be employed to study the unknown function of EMBPPR genes. By this method, we showed that EMB976 is required for splicing of chloroplast clpP1 intron 2 and ycf3 intron 1.

Keywords: Arabidopsis, EMB PPR genes, Cosuppression, PTGS, RDR6

\section{Background}

It is a common phenomenon that knockout mutants of plant essential genes display embryo or seedling lethal phenotype [1]. In the Arabidopsis genome, there are about 510 EMBRYO-DEFECTIVE $(E M B)$ genes.

*Correspondence: huangweihua@shnu.edu.cn

Shanghai Key Laboratory of Plant Molecular Sciences, College of Life

Sciences, Shanghai Normal University, Shanghai 200234, China
Among them, 34 genes belong to the pentatricopeptiderepeat (PPR) gene family $[1,2]$. PPR proteins characterized by tandem arrays of a degenerate 35 -amino-acid repeat (PPR motif) are conserved in all eukaryotes and extremely expanded in higher plants. It has been known that over 450 PPR proteins are present in Arabidopsis and divided into two subfamilies, designated P and PLS, according to the characteristics of the PPR motifs [3]. Most PPRs are involved in regulation of organelle gene

(c) The Author(s) 2020. This article is licensed under a Creative Commons Attribution 4.0 International License, which permits use, sharing, adaptation, distribution and reproduction in any medium or format, as long as you give appropriate credit to the original author(s) and the source, provide a link to the Creative Commons licence, and indicate if changes were made. The images or other third party material in this article are included in the article's Creative Commons licence, unless indicated otherwise in a credit line to the material. If material is not included in the article's Creative Commons licence and your intended use is not permitted by statutory regulation or exceeds the permitted use, you will need to obtain permission directly from the copyright holder. To view a copy of this licence, visit http://creativeco mmons.org/licenses/by/4.0/. The Creative Commons Public Domain Dedication waiver (http://creativecommons.org/publicdomain/ zero/1.0/) applies to the data made available in this article, unless otherwise stated in a credit line to the data. 
expression, including RNA cleavage, splicing, editing and stability, via directly binding to their RNA targets [4]. A set of EMB PPRs have been reported to be required in RNA processing of the chloroplast housekeeping genes. For example, EMB2261 is specifically involved in chloroplast rps14 mRNA editing [5]. EMB2750 (PPR2) is specifically involved in chloroplast 23S rRNA processing [6]. EMB3140 (PDM3) is specifically involved in chloroplast $\operatorname{trn} A$ and clpP1 intron1 splicing [7]. EMB2279/SOT5 is specifically involved in chloroplast $r p l 2$ and $t r n K$ intron splicing [8]. EMB2654 and atPPR4 are coordinately and specifically involved in trans-splicing of plastid rps 12 intron1 $[9,10]$. And more recently EMB976/PDM4 is reported to be specifically involved in chloroplast group II intron splicing including $c l p P 1$ and $y c f 3$ [11]. Since the targets of these PPRs are usually housekeeping genes in chloroplasts, it is understandable that knockout mutants of these PPRs are embryonically lethal. Thus, generation of weak allele mutants, viable knockdown lines or partially complementary lines of EMB PPRs is required for their functional analysis. Currently, techniques such as antisense, RNAi, artificial microRNA are employed to make knockdown lines [12, 13]. Complementation of emb2654 heterozygous lines with a cDNA carrying the WT coding sequences under the control of the seed-specific ABSCISIC ACID-INSENSITIVE3 (ABI3) promoter is employed to generate the partially complementary lines [10]. In addition, CRISPR/CAS9 techniques are also widely used for constructing knockout or knockdown mutants [14, 15]. However, these techniques are sometimes not efficient to get wanted transgenic plants. For example, small interfering RNA (siRNA) and artificial microRNA (amiRNA), which are about 21-22 nucleotide (nt), often target more than one endogenous genes if the gene sequence is very conserved among its gene family [13]. And the knockdown lines generated by RNAi or artificial microRNA sometimes display very mild phenotypes, which is not helpful for phenotypical study. Constructing partially complementary lines is a time-consuming process due to making transgenic plants in heterozygotes at first [10]. CRISPR/CAS9 techniques usually cause off-target effects, and identification of the genome-edited lines is relatively costly [14].

The gene cosuppression often occurs when a homologous coding sequence is overexpressed in plants [16-18]. This phenomenon is controlled by the post transcriptional gene silencing (PTGS) pathway [12], which is initiated by the conversion of single-stranded RNAs (over accumulated aberrant RNAs) into double-stranded RNAs by RNA-dependent RNA polymerase 6 (RDR6). The double strand RNAs are subsequently sliced into 21-22 nt small interfering RNAs (siRNA) by dicer-like 2 (DCL2) or DCL4. siRNAs are loaded into the RNA-induced silencing complex (RISC) to cleave the target RNAs [19, 20]. The PTGS pathway is well conserved among eukaryotes. Based on this pathway, antisense, RNA interfering (RNAi) and artificial microRNA techniques are developed and widely used to construct knockdown lines for a lot of model organisms to facilitate their gene function studies [12].

Here, we reported a method that can efficiently make cosuppression lines via overexpressing an appropriately truncated PPR CDS in the WT background. In addition, we dissected the biological function of a PPR protein, EMB976, in Arabidopsis using this method.

\section{Results}

\section{Overexpression of the mutated EMB2279/SOT5} and EMB2654 CDS in WT leads to RDR6-dependent gene silencing

We previously reported that a weak allele of emb2279-2/ sot 5 mutant exhibits a virescent phenotype, which is caused by a point mutation that significantly reduces splicing efficiency of the seventh intron of SOT5 and generates two additional mRNA variants [8]. The smallest transcript that lacks 22-base pairs (bp) at the $3^{\prime}$ end of the seventh exon is predicted to produce a truncated SOT5 protein with only 6 PPR motifs, named SOT5-m1 (Fig. 1), whereas the largest transcript that contains the seventh intron is predicted to produce a mutated protein with 10 PPR motifs, named SOT5-m2 (Fig. 1). While the wild type SOT5 encodes a protein with 11 PPR motifs (Fig. 1 and Table 1). To test whether the two predicted proteins are functional or not in plants, we cloned SOT5$m 1$ and SOT5-m2 CDS and transformed them under the control of the cauliflower mosaic virus $35 \mathrm{~S}$ promoter into WT plants. We obtained 30 and 42 positive $\mathrm{T}_{1}$ transformants for 35S:SOT5-m1 and 35S:SOT5-m2 constructs, respectively. Our results showed that $80 \%$ of the 35S:SOT5-m1 transgenic lines exhibited severe leaf chlorosis at the early growth stage, and these leaves were gradually turned into pale green later; and $48 \%$ of 35S:SOT5- $m 2$ transgenic lines displayed relatively mild leaf chlorosis and virescence (Table 1 and Fig. 2a). This chlorosis and virescent phenotype was similar to that of the sot 5 mutant. Thus, we suspected that the transgenic lines with chlorosis were the cosuppression lines, in which expression of the endogenous SOT5 was silenced. Then, we analyzed expression levels of SOT5 in $\mathrm{T}_{1}$ transformants with chlorosis. Indeed, RT-PCR and quantitative PCR (qPCR) analyses showed that the level of the endogenous SOT5 transcripts (En-SOT5), detected by the specific primer pair spanning $5^{\prime} \mathrm{UTR}$ (the red arrows highlighted on EMB2279/SOT5 cDNA in Fig. 1), was significantly decreased in 35S:SOT5-m1/Col-0 transgenic line 2 and line3 (less than $40 \%$ of the WT expression 


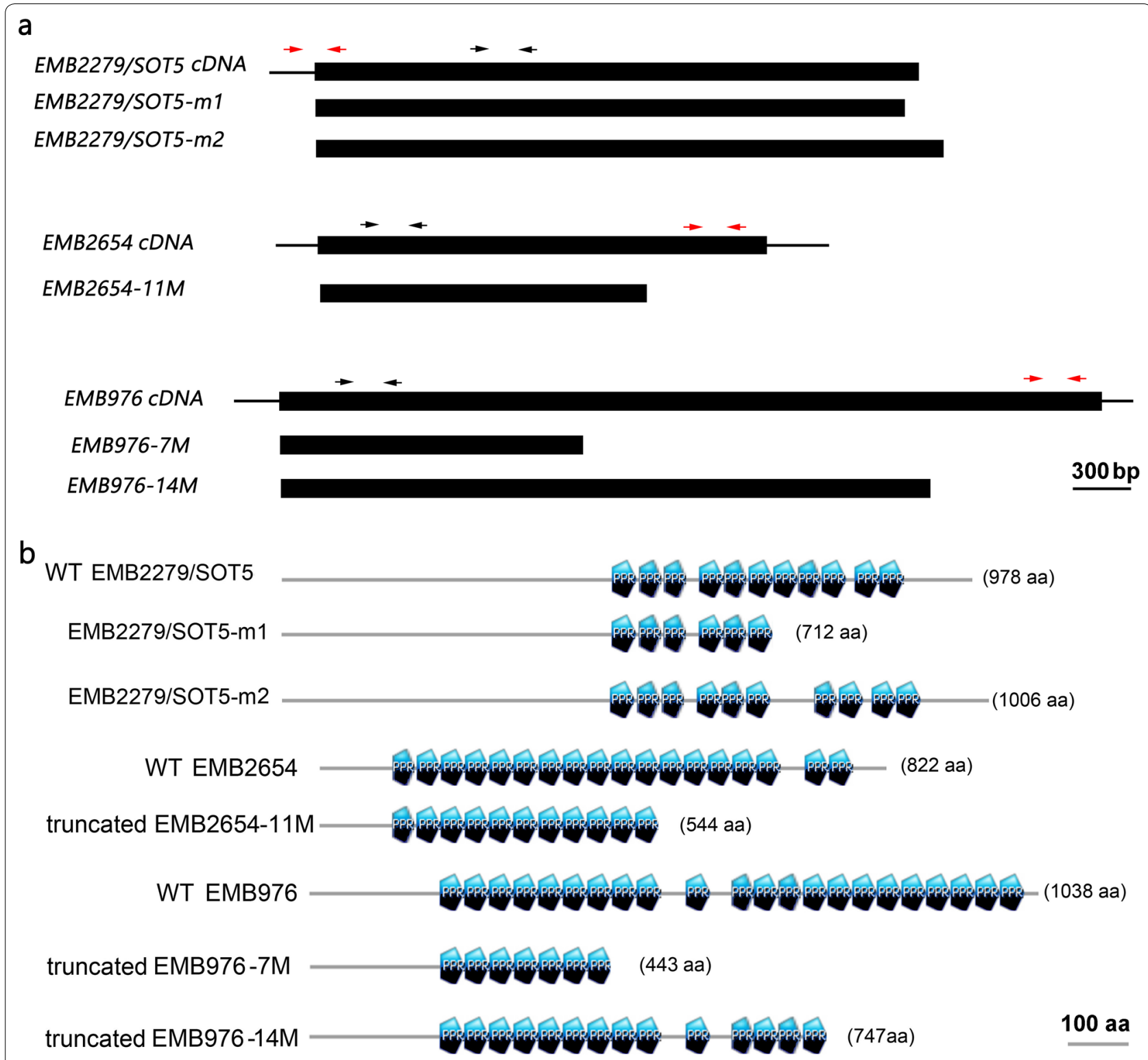

Fig. 1 Schematic diagram of the EMBPPR CDS constructs made in this study and their coding proteins. a Schematic diagram of the EMB PPR CDS constructs. The EMB PPR CDS is indicated by black box. The UTR regions are indicated by black lines. The black arrows show the position of the primer pair to detect the total genes (including endogenous and transgenes). The red arrows show the position of the primer pair to detect the endogenous genes. Bar $=300 \mathrm{bp}$. $\mathbf{b}$ Schematic diagram of the corresponding EMB PPR proteins. The protein structures were predicted by PROSITE (https://prosite.expasy.org/). Pentagons mean PPR motifs in each construct. The amino acid (aa) numbers indicate the size of WT or truncated PPR proteins. $\mathrm{Bar}=100 \mathrm{aa}$

level), whereas the level of total SOT5 transcripts (including endogenous and exogenous SOT5 in transgenic lines), detected by the primer pair spanning intron 7 (Fig.2b) or by the primer pair in CDS (black arrows highlighted on EMB2279/SOT5 cDNA in Fig. 1) was significantly increased (Fig.2c), compared with that in non-transgenic WT plants. Consistently, in 35S:SOT5-m2/Col-0 transformants, the expression level of endogenous SOT5 was also significantly decreased (less than $60 \%$ of the WT expression level, Fig. 2b, c), while the content of total SOT5 transcripts was significantly increased (Fig. 2d). Meanwhile, splicing efficiency of the plastid rpl2 gene, a SOT5 target, was dramatically reduced in these transformants (Fig. 2b, e). In contrast, splicing efficiency of the plastid atpF gene, a non-target gene of SOT5, was not altered in all transgenic plants (Fig. 2b, e). These results 
Table 1 The cosuppression phenotype and frequency of transgenic plants expressing various constructs in WT

\begin{tabular}{|c|c|c|c|c|c|c|c|}
\hline $\begin{array}{l}\text { Plamid } \\
\text { construct }^{\mathrm{a}}\end{array}$ & $\begin{array}{l}\text { Length } \\
\text { of encoding } \\
\text { protein (aa) }\end{array}$ & $\begin{array}{l}\text { Number } \\
\text { of PPR } \\
\text { motifs }\end{array}$ & $\begin{array}{l}\text { Phenotype } \\
\text { of cosuppression }\end{array}$ & $\begin{array}{l}\text { Number } \\
\text { of total } T_{1} \\
\text { transformants }\end{array}$ & $\begin{array}{l}\text { Number of } \mathrm{T}_{1} \\
\text { transformants } \\
\text { with visible } \\
\text { chlorosis }\end{array}$ & $\begin{array}{l}\text { Frequency } \\
\text { of cosuppression }^{b}\end{array}$ & $\begin{array}{l}\text { Severity } \\
\text { of chlorosis } \\
\text { in cosuppression } \\
\text { lines }\end{array}$ \\
\hline SOT5 & 978 & 11 & $\begin{array}{l}\text { Yellow inflores- } \\
\text { cence and } \\
\text { cauline leaves }\end{array}$ & 35 & 20 & $57 \%$ & Very mild \\
\hline SOT5-ml & 712 & 6 & $\begin{array}{l}\text { Albino young } \\
\text { leaves at seed- } \\
\text { ling stage }\end{array}$ & 30 & 24 & $80 \%$ & Strong \\
\hline SOT5-m2 & 1006 & 10 & $\begin{array}{l}\text { Partial albino } \\
\text { leaves at seed- } \\
\text { ling stage }\end{array}$ & 42 & 20 & $48 \%$ & Mild \\
\hline EMB2654 & 822 & 18 & ND & ND & ND & ND & ND \\
\hline EMB2654-11M & 550 & 11 & $\begin{array}{l}\text { Chlorosis leaves at } \\
\text { seedling stage }\end{array}$ & 47 & 47 & $100 \%$ & Mild \\
\hline EMB976 & 1038 & 22 & ND & ND & ND & ND & ND \\
\hline EMB976-7M & 443 & 7 & WT-like & 13 & 0 & $0 \%$ & ND \\
\hline EMB976-14M & 747 & 14 & $\begin{array}{l}\text { Yellow young } \\
\text { leaves at seed- } \\
\text { ling stage }\end{array}$ & 8 & 4 & $50 \%$ & Mild \\
\hline
\end{tabular}

${ }^{a}$ The pGWB2 plasmid containing the mutated or truncated CDS indicated by the name

b Cosuppressed $\mathrm{T}_{1}$ tranfromants/total $\mathrm{T}_{1}$ transformants

ND not determined

indicate that overexpression of the mutated SOT5 CDS in WT leads to suppression of the endogenous gene expression, probably through the post transcriptional gene silencing (PTGS, also known as sense cosuppression) pathway.

To confirm this hypothesis, we transformed 35S:SOT5$m 1$ and 35S:SOT5-m2 constructs into the rdr6-11 mutant. RDR6 is a key component in the PTGS pathway and the rdr6-11 mutant presented elongated and curled downward leaves (Fig. 3a) [21, 22]. It has been demonstrated that overexpression of homologous genes in this mutant was not able to trigger the PTGS pathway [21-23]. We obtained twenty-five 35S: SOT5-m1/rdr6 and eighteen 35S: SOT5-m2/rdr6 T1 transformants, and found that none of the transformants exhibited the chlorosis phenotype (Fig. 3a). RT-PCR analysis showed that the endogenous SOT5 transcript level was not decreased, although the total SOT5 mRNA level was significantly increased in the transformants (Fig. 3b-d). Consequently, no splicing defect of plastid $r p l 2$ was detected in these transformants (Fig. 3e). Taken together, our results indicate that overexpression of the mutated SOT5 CDS in the WT background leads to PTGS in a RDR6-dependent manner.

We then asked whether the above result could be repeated with other $E M B P P R$ genes. To address this question, we overexpressed a truncated CDS of
EMB2654 (EMB2654-11M) which encodes a truncated protein with only 11 PPR motifs into the WT background (Fig. 1 and Table 1). While the wild type EMB2654 has 18 PPR motifs. It has been reported that EMB2654, a P family PPR protein, was required for trans-splicing of the plastid gene rps12 intron 1 [10]. Interestingly, all the $47 \mathrm{~T}_{1}$ transgenic lines exhibited leaf chlorosis (Table 1 and Fig. 4a), indicating the silence of endogenous EMB2654. RT-PCR analysis showed that expression of the endogenous EMB2654 was down-regulated (about 20\% of the WT expression level) while the total mRNA of EMB2654 was significantly increased in these transformants (Fig. 4b-d). Indeed, the splicing efficiency of rps 12 intron 1 was significantly decreased in the cosuppression lines, while the splicing efficiency of the non-target gene, clpP1 intron2 was not significantly decreased (Fig. 4e). However, when the truncated EMB2654-11M CDS was overexpressed in $r d r 6-11$, no leaf chlorosis was observed among 30 transformants (Fig. 4f). RT-PCR results showed that the endogenous EMB2654 mRNA was not decreased, and the total EMB2654 mRNA was significantly increased in these transformants (Fig. 4gi). Consistently, splicing efficiency of the rps12 intron 1 was not decreased in these transformants (Fig. 4j). Thus, these results confirm that overexpression of the truncated EMB2654 CDS in the WT background leads to PTGS in a RDR6-dependent manner. 
a

35S:SOT5-m2/Col-0

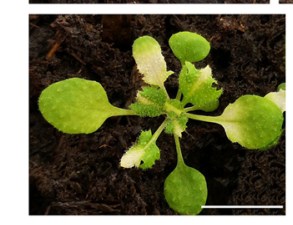

C
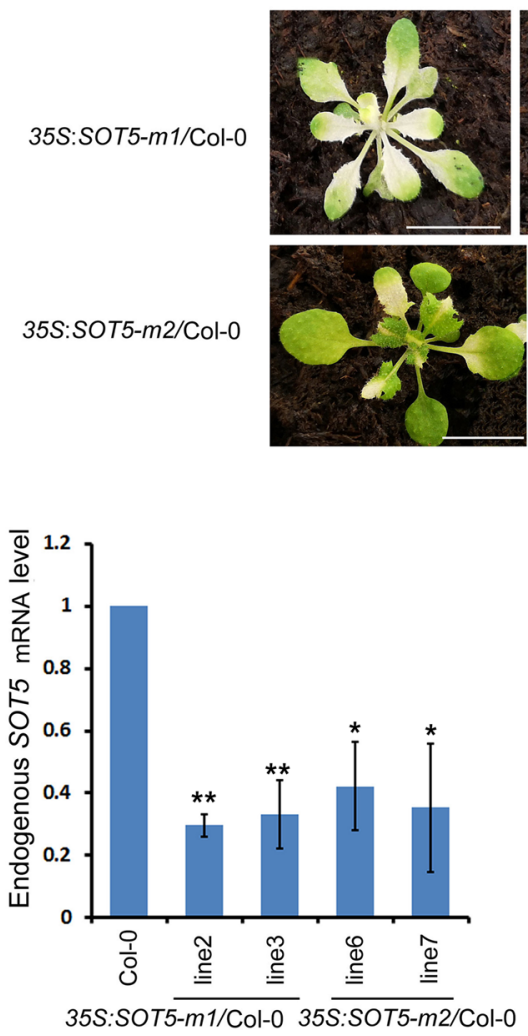
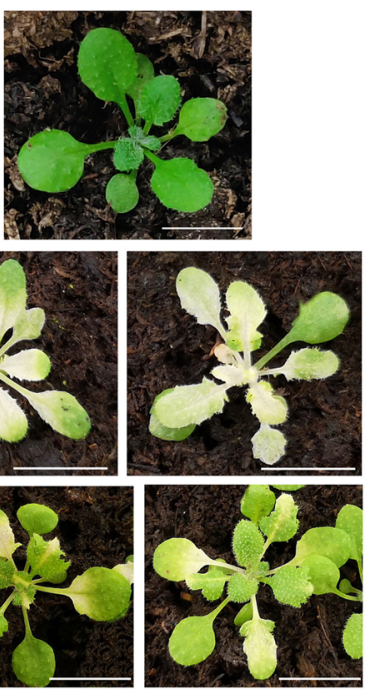

d

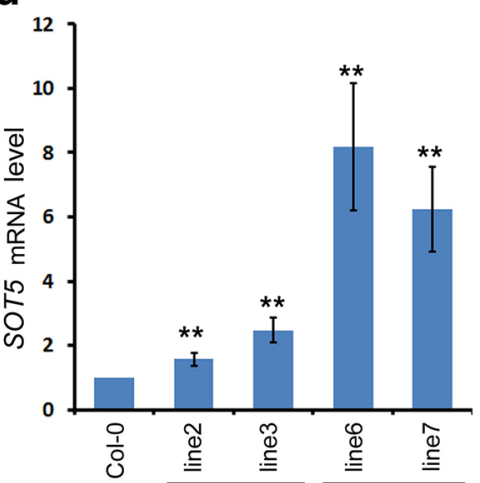

35S:SOT5-m1/Col-0 35S:SOT5-m2/Col-0 b

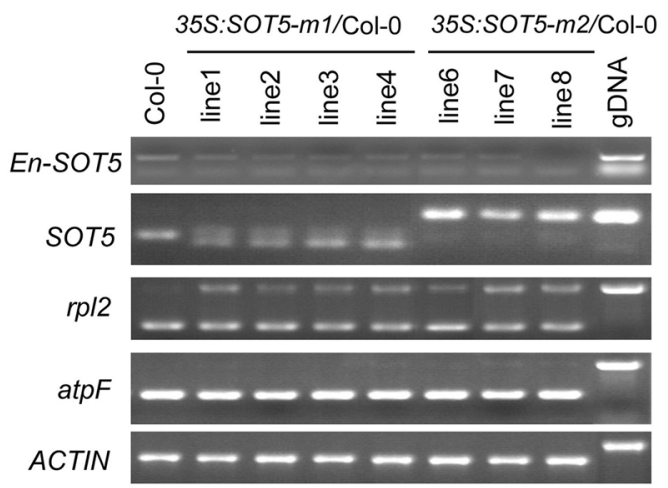

e

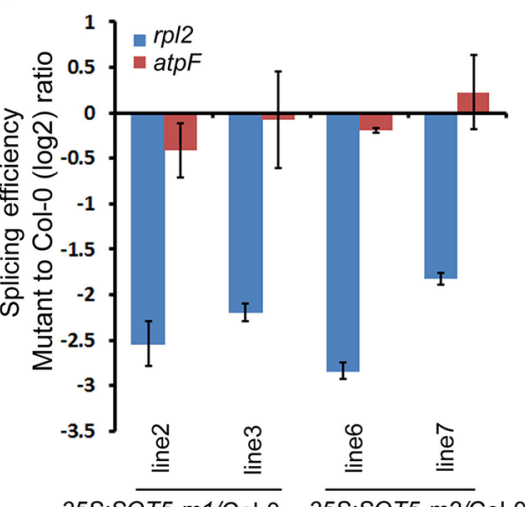

Fig. 2 The phenotypes of transgenic plants overexpressing the mutated SOT5 CDS in WT background. a Chlorosis leaf phenotype of transgenic plants overexpressing SOT5-m1 and SOT5-m2 constructs in WT background (bar = $1.5 \mathrm{~cm}$ ). b RT-PCR analysis of expression levels of the endogenous SOT5, total SOT5 (including endogenous SOT5 and transgene), and two plastid genes in the typical cosuppression lines. $\mathbf{c}$ RT-qPCR analysis of the endogenous SOT5 transcript level in the cosuppression lines. $\mathbf{d}$ RT-qPCR analysis of the total SOT5 transcript level in the cosuppression lines. $\mathbf{e}$ Splicing efficiency of plastid $r p / 2$ and atpF (as a control) in the cosuppression lines. For RT-qPCR, the values are means of three technique replicates (bars indicate SD). Asterisks indicate significant differences between wild type (WT) and transgenic plants (Student's t test, *, P $<0.05$ and ${ }^{* *}$, $\mathrm{P}<0.01)$. Two to three $\mathrm{T1}$ transformants were analyzed for RT-PCR and RT-qPCR

\section{Functional analysis of EMB976 via its cosuppression lines}

EMB976 is a functionally unknown PPR protein, which belongs to P subfamily containing 22 PPR motifs and is predicted to be localized in chloroplasts. Its knockout mutant has been demonstrated to be embryonically lethal [2]. To study its physiological role in plant growth, we constructed two plasmids named EMB976$7 M$ and EMB976-14M, which encode the truncated protein with 7 and 14 PPR motifs, respectively (Fig. 1 and Table 1), and transformed them into the WT background. Our results showed that four of eight 35S:EMB976-14M/Col-0 $\mathrm{T}_{1}$ transformants displayed virescent leaves (Fig. 5a and Table 1), whereas all of the thirteen 35S:EMB976-7M//Col-0 transformants had the same phenotype as WT (Table 1). To confirm whether gene silencing occurred in 35S:EMB976-14M/Col-0, we checked expression levels of the endogenous $E M B 976$ in the transformants with the virescent phenotype. As expected, the endogenous EMB976 was significantly down-regulated (about $40 \%$ of the WT expression level) while the total $E M B 976$ mRNA was significantly increased in these transformants (Fig. 5b, c), indicating that the phenotype of the transgenic lines is caused by the silencing of $E M B 976$. Since the P family PPR proteins were often involved in organelle RNA stability and 


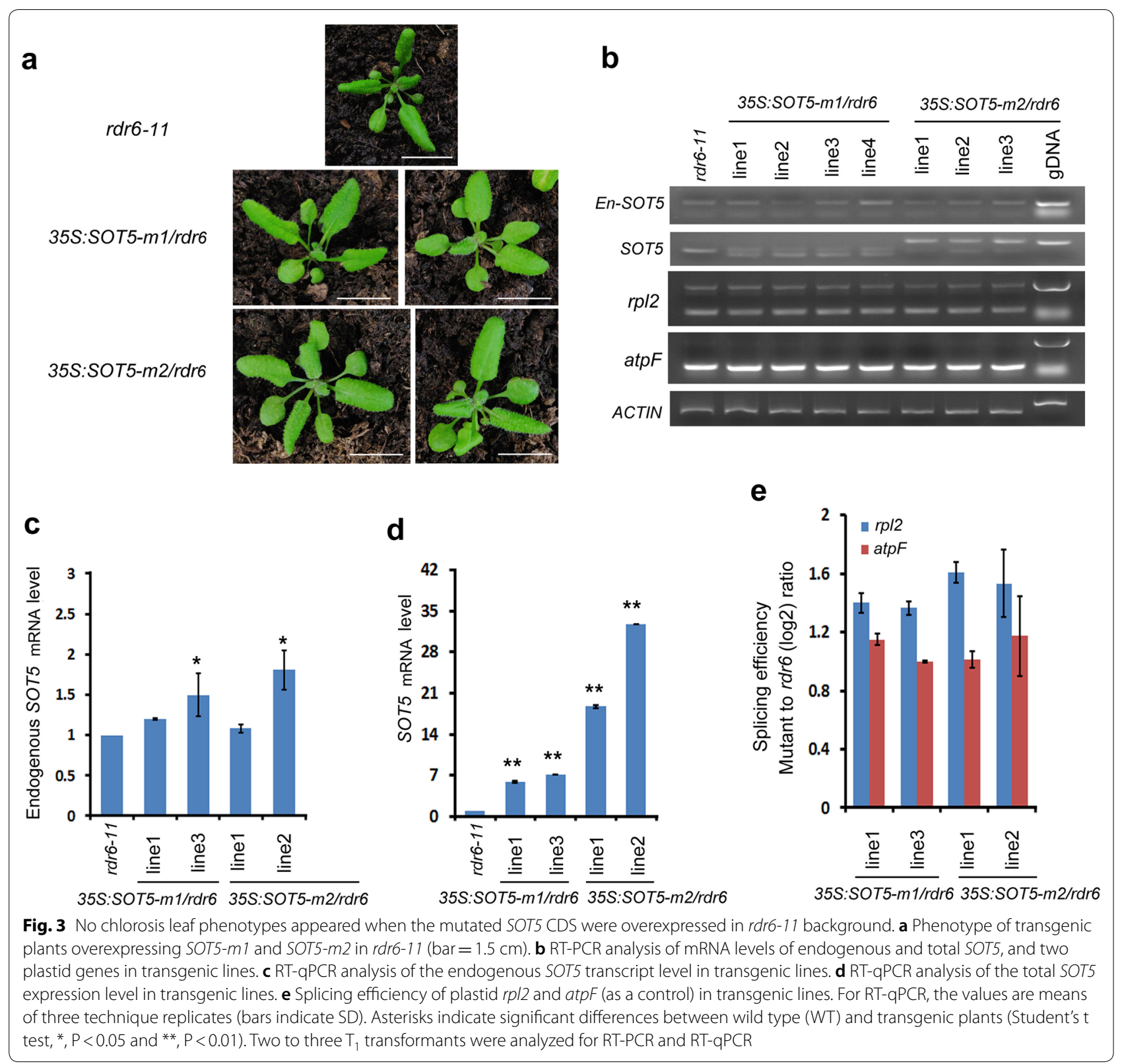

(See figure on next page.)

Fig. 4 The phenotypes of transgenic plants overexpressing the truncated EMB2654-11M are dependent on RDR6. a Overexpression of the truncated EMB2654-11M in WT background leads to chlorosis phenotype (bar $=1.5 \mathrm{~cm}$ ). b RT-PCR analysis of the endogenous and total EMB2654 transcripts level in the cosuppression lines. c RT-qPCR analysis of the endogenous EMB2654 transcripts level in the cosuppression lines. $\mathbf{d}$ RT-qPCR analysis of the total EMB2654 transcripts level in the cosuppression lines. e RT-qPCR analysis of the splicing efficiency of plastid rps 12 intron 1 in the cosuppression lines. f Phenotype of transgenic plants overexpressing EMB2654-11M in $r d r 6-11(\mathrm{bar}=1.5 \mathrm{~cm}) . \mathbf{g}$ RT-PCR analysis of the endogenous and total EMB2654 transcripts level in the transformants. h RT-qPCR analysis of the endogenous EMB2654 transcripts level in the transformants. i RT-qPCR analysis of the total EMB2654 transcript level in transgenic lines. j Splicing efficiency of plastid $r p s 12$ intron 1 in the transformants. For RT-qPCR, the values are means of three technique replicates (bars indicate SD). Asterisks indicate significant differences between wild type (WT) and transgenic plants (Student's t test, ${ }^{*}, P<0.05$ and ${ }^{* *}, P<0.01$ ). Two to three $T_{1}$ transformants were analyzed for RT-PCR and $q R T-P C R$ 
a

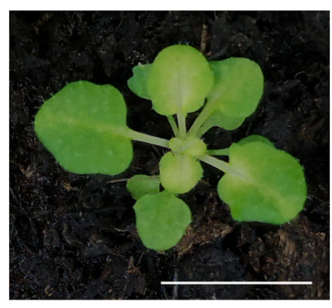

35S:EMB2654-11M/Col-0

C

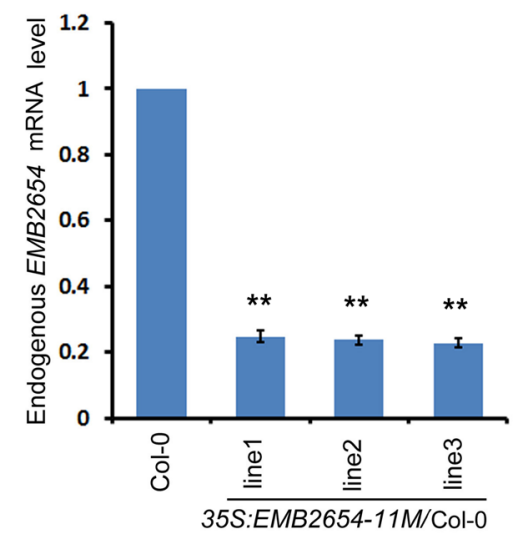

d

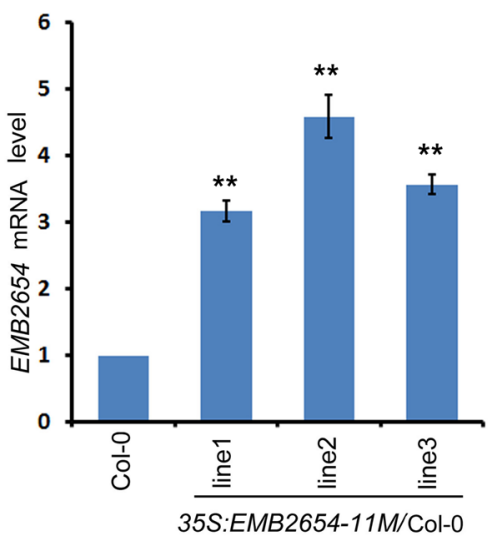

b

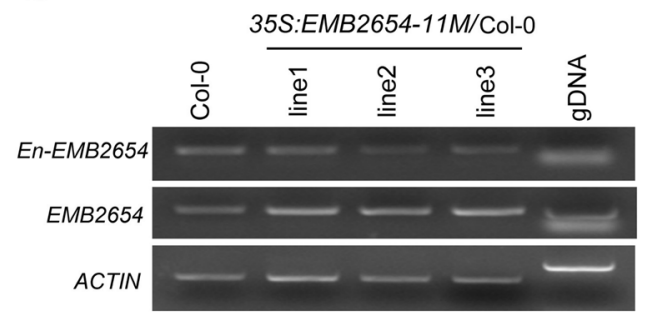

e

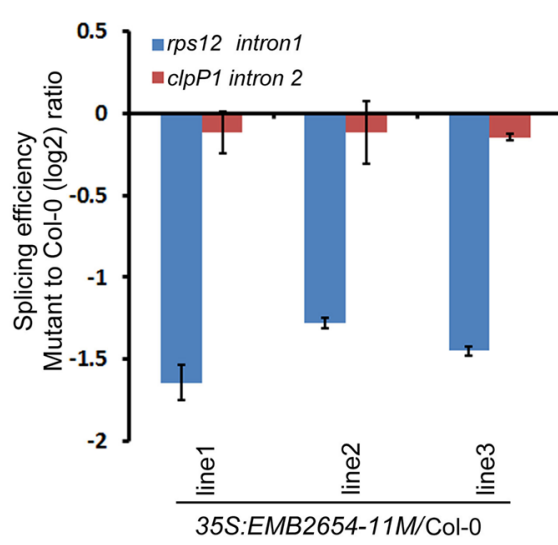

\section{f}
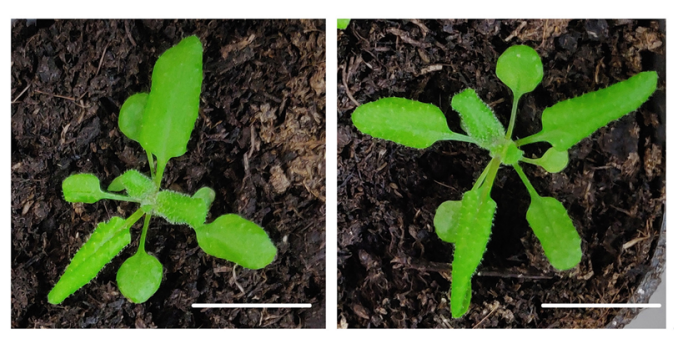

35S:EMB2654-11M/rdr6

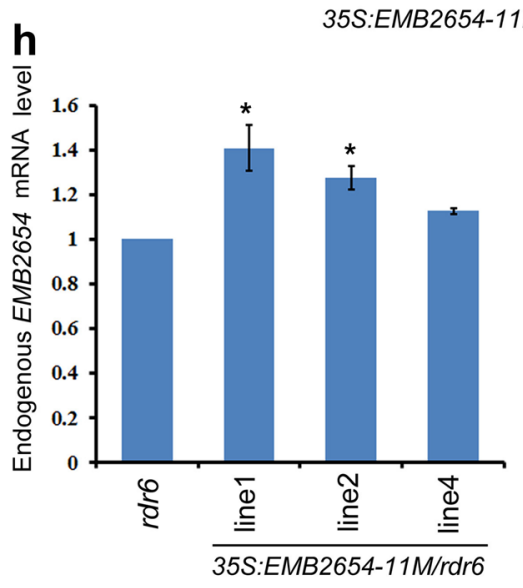

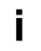

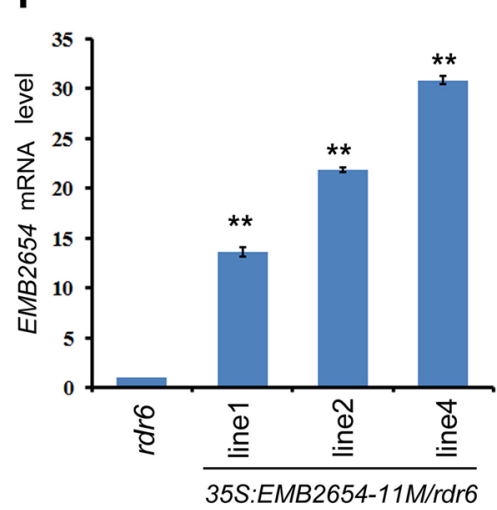

j

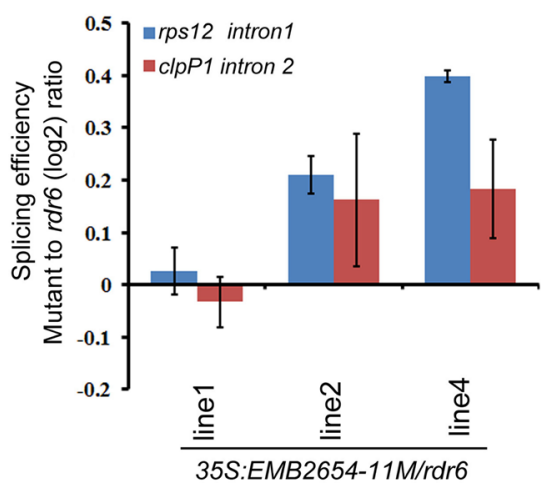


(See figure on next page.)

Fig. 5 Functional analysis of EMB976 via overexpressing truncated EMB976 CDS in WT. a Yellow leaf phenotype of $T_{1}$ transgenic plants overexpressing the truncated EMB976-14M (bar $=1.5 \mathrm{~cm})$. b RT-qPCR analysis of mRNA levels of the endogenous EMB976 in transgenic lines. $\mathbf{c}$ RT-qPCR analysis of mRNA levels of total EMB976 in transgenic lines. $\mathbf{d}$ RT-PCR analysis of precursor transcripts of chloroplast genes with specific primers. The number after the decimal point indicates the intron of the gene. e Splicing efficiency of $c / p P 1$ intron 2 and ycf3 intron 1 in the cosuppression lines. $\mathbf{f}$ Phenotype of $\mathrm{T}_{1}$ plants overexpressing truncated EMB976-14M in rdr6-11 (bar $\left.=1.5 \mathrm{~cm}\right) . \mathbf{g}$ RT-PCR analysis of mRNA levels of endogenous EMB976, total EMB976, clpP1 and ycf3 precusors in the transformants. $\mathbf{h}$ RT-qPCR analysis of mRNA levels of endogenous EMB976 in the transformants. i RT-qPCR analysis of mRNA levels of total EMB976 in the transformants. $\mathbf{j}$ RT-qPCR analysis of splicing efficiency of plastid clpP1 intron 2, ycf3 intron 1 and ycf3 intron 2 in the transformants. For RT-qPCR, the values are means of three technique replicates (bars indicate SD). Asterisks indicate significant differences between wild type (WT) and transgenic plants (Student's t test, ${ }^{*}, \mathrm{P}<0.05$ and ${ }^{* *}, \mathrm{P}<0.01$ ). Two to three T1 transformants were analyzed for RT-PCR and RT-qPCR

splicing, we further examined the intron splicing of chloroplast genes in these 35S:EMB976-14M/Col-0 cosuppression lines. Indeed, RT-PCR analysis showed that the precursors of $n d h A, c l p P 1$ intron 2 and $y c f 3$ intron 1 were significantly and specifically accumulated in these lines (Fig. 5d). qPCR analysis showed that the splicing efficiency of $c l p P 1$ intron 2 and $y c f 3$ intron 1 was significantly and specifically decreased in these lines, compared with that of $y c f 3$ intron 2 (Fig. 5e), suggesting chloroplast clpP1 intron 2 and $y c f 3$ intron 1 were the possible targets of EMB976. However, further experiments are required for verification of the results. Again, no yellow young leaves were observed among the transformants when EMB976-14M was overexpressed in $r d r 6-11$ (Fig. 5f). This result was consistent with that of RT-PCR analysis. In these transformants, the endogenous EMB976 transcripts was not significantly decreased while the total EMB976 transcripts was strongly increased (Fig. $5 \mathrm{~g}-\mathrm{i}$ ). Consistently, the splicing efficiency of $c l p P 1$ intron 2 and $y c f 3$ intron 1 was not decreased, compared with that of $y c f 3$ intron 2 (Fig. 5j). Thus, we conclude that gene silencing triggered by overexpession of truncated $E M B$ genes is dependent on RDR6.

\section{Discussion}

Overexpressing mutated EMB PPRs CDS in WT background leads to specific silencing of the endogenous genes

Since it's hard to get knockout mutants for $E M B$ genes, obtaining weak alleles or constructing viable knockdown lines of $E M B$ genes is valuable for their functional study. For example, by characterization its weak allele, the PPR protein EMB2279 was disclosed to be specifically involved in chloroplast trnk and $r p l 2$ intron splicing [8]. And by the help of its partial complementation line, EMB2654 was revealed to be specifically involved in trans-splicing of chloroplast rps12 intron1 [10].

In this study, we found a simple way to make cosuppression lines of $E M B P P R$ gene by overexpressing its appropriately truncated CDS fragment in WT background. In the cosuppression lines, the over-accumulated transgene's transcripts are converted into double-stranded RNAs by RDR6. Then the double strand
RNAs are subsequently sliced into $21-22$ nt siRNA by dicer-like 2 (DCL2) or DCL4. siRNAs are loaded into the RNA-induced silencing complex (RISC) to cleave the target RNAs [20]. The silenced gene is specifically targeted by its exogenous (transgene) sequence in the cosuppression lines. In our study, the sequence of transgene is long enough (more than or close to $2 / 3$ of WT full length CDS, Table 1), theoretically, the targeting is more specific than that of siRNA or amiRNA. As expected, by RT-qPCR analysis, we found the expression level of EMB2654 or $E M B 976$ was not significantly decreased in 35:SOT5-m1/ Col-0 or 35:SOT5-m2/Col-0 cosuppression lines (Additional file 1: Fig. S1a, b). The expression level of EMB2654 was not significantly decreased in 35S:EMB976-14M/ Col-0 cosuppression lines and vice versa (Additional file 1: Fig. S1c, d). These results indicate that the targeting is very specific by cosuppression.

Besides the $E M B$ genes encoding chloroplasts localized PPR proteins, we also overexpressed an alternative splicing variant of $E M B 2784 / P R P L 4$, which encodes a plastid ribosomal protein L4 into WT background. Compared with the major splicing variant PRPL4 CDS, the gPRPL4 CDS retains its unique intron with a premature termination coden (PTC) and leads to a truncated PRPL4 protein. Interestingly, among $9 \mathrm{~T}_{1}$ transformants, there were 6 transformants exhibited chlorosis leaves (Additional file 2: Fig. S2a). RT-PCR analysis showed that in these transformants with chlorosis leaves, the endogenous PRPL4 was significantly decreased (asterisk in Additional file 2: Fig. S2b) and the exogenous gPRPL4 was increased (arrowhead Additional file 2: Fig. S2b). Meanwhile, RTqPCR analysis confirmed that PRPL4 was specifically decreased in the 35S:gPRPL4/Col-0 cosuppression lines when compared with the expression level of PRPL28 (PLASTID RIBOSOMAL PROTEIN L28, Additional file 2: Fig. S2c, d). These results indicated that overexpression of a mutated CDS of PRPL4 in WT also leads to cosuppression with a high frequency. Taken together, it seems that this method is universal and robust for constructing knockdown lines for the chloroplast localized EMB proteins. It is likely that dysfunction of the chloroplast-localized proteins often leads to visible chlorosis phenotypes, 


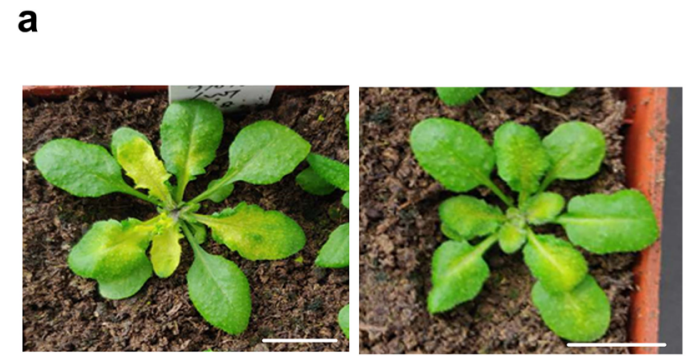

35S:EMB976-14M/Col-0

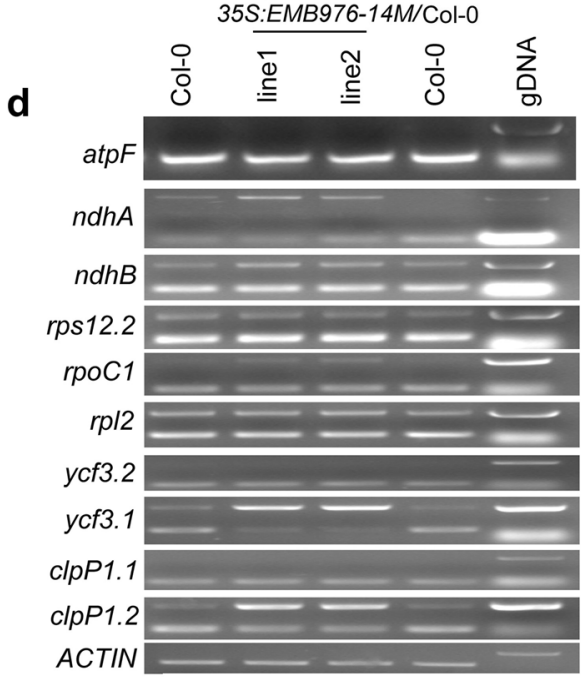

f

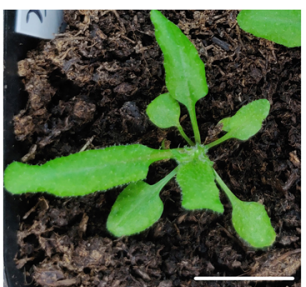

35S:EMB976-14M/rdr6

h

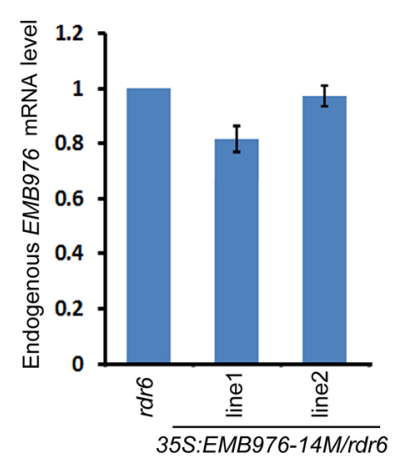

b

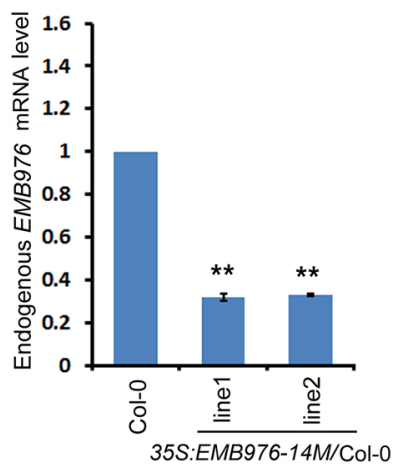

e
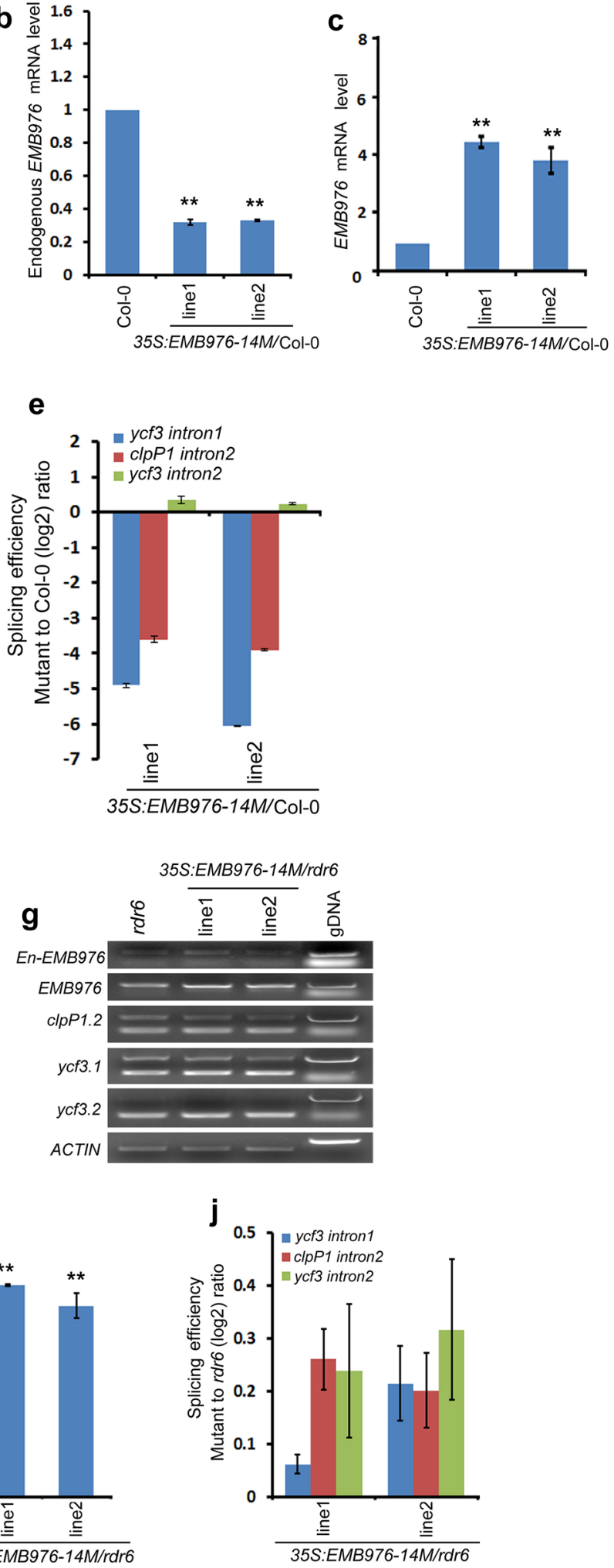
which makes us easy to know whether the target gene is silenced in transgenic lines. In the future, we will test more genes using this method and try to work out an optimal experimental system to construct cosuppression lines efficiently for $E M B$ genes in Arabidopsis.

\section{The length of truncated PPR CDS is critical for obtaining cosuppression lines with strong mutant phenotype efficiently}

We found that cosuppression hardly occurred in the transgenic lines if the length of truncated PPR CDS overexpressed in WT plants was less than half of the full PPR CDS, such as EMB976-7M (Table 1). This result is consistent with the report that overexpression of exogenous Chalcone Synthase (Chs) with a premature translational termination codon (PTC) significantly decreased the frequency of cosuppression, compared with WT Chs in Petunia [24]. And Mallory et al. [23] also reported that when the transgene sequence only shared $557 \mathrm{nt}$ of homology with the endogenous AGO1 mRNA, the cosuppression frequency was significantly lower than that using the transgene sequence shares more than $3 \mathrm{~kb}$ of homology with the endogenous AGO1 mRNA. The transcripts of transgene with PTC were probably degraded by the nonsense-mediated mRNA decay (NMD) pathway, which can recognize and degrade aberrant transcripts harboring PTC, and thereby prevent the production of truncated proteins which might be deleterious [25]. However, if the PTC occurrs close to the $3^{\prime}$-UTR, the mRNA will escape from the NMD pathway, and thus can accumulate and trigger the RDR6-dependent PTGS pathway $[26,27]$. So, theoretically, the full length of CDS will lead to a highest cosuppression frequency. However, we found that when the full length of SOT5 was overexpressed in WT background, only very mild chlorosis (cosuppression) phenotypes were observed at late developmental stage (Additional file 3: Fig. S3), although the cosuppression frequency is high enough (Table 1). In contrast, overexpression of the truncated (more than 2/3 of WT full length CDS, Table 1) or mutated SOT5 CDS in WT led to a high cosuppression frequency and the strong mutant phenotype. It is likely that the absence of endogenous SOT5 protein due to PTGS can be partially compensated by the truncated or mutated SOT5 protein in the cosuppression lines. These results are consistent with the report that the developmental defects of cosuppressed plants were more pronounced in a non-functional AGO1 protein transformants than in a functional AGO1 protein transformants [23]. Thus, we suggest that the appropriate truncation of PPR CDS is critical for constructing cosuppression lines with strong mutant phenotype efficiently.
EMB976 is required for splicing of chloroplast ClpP1 intron 2 and $y c f 3$ intron 1

We investigated the role of functionally unknown EMB976 in plant growth by overexpressing the truncated EMB976-14M CDS in WT. Our data showed that the 35S: EMB976-14M/Col-0 cosuppression lines displayed a leaf virescent phenotype and splicing efficiency of clpP1 intron 2 and $y c f 3$ intron 1 was significantly deceased in these cosuppression lines. ClpP1 is a core component of $\mathrm{ClpP}$ protease complex, which plays critical roles in chloroplast protein homeostasis $[28,29]$. Ycf3 is involved in assembly of photosynthetic complex I, which is a key complex in light reaction of photosynthesis [30]. The absence of these critical proteins due to the decreased mature RNAs may explain why the knockout mutant (emb976) is embryonically lethal. Interestingly, a recent work showed that EMB976/PDM4 is involved in splicing of group II introns and rRNA processing in chloroplasts [11]. They found the precursors of the chloroplast $n d h A$, pet $B$, $y c f 3$, petD and $\operatorname{clpP} 1$ were present and accumulated in a high level in the $p d m 4$ knockout mutant but absent in the wild type, which is consistent with our results. The role of EMB976 for group II intron splicing might be independent on its role for rRNA processing. It's probable that rRNA processing defect is an indirect effect of absence of the chloroplast ClpP protease complex since it was reported that in the weak allele, clpR4-3 mutant, the plastid rRNA processing was also defective [31].

\section{Conclusions}

Overexpression of an appropriately truncated $E M B P P R$ CDS in WT leads to generation cosuppression lines with strong mutant phenotype efficiently, and this method can be employed to study the unknown function of $E M B P P R$ genes. By this method, we found that EMB976 is required for intron splicing of plastid $c l p P 1$ and $y c f 3$.

\section{Materials and methods \\ Plant materials and growth conditions}

The Arabidopsis ecotype Columbia-0 (Col-0) was used as WT in this study. The $r d r 6-11$ mutant used in this study was previously described [22]. Seeds were surfacesterilized by $75 \%$ ethanol and stratified at $4{ }^{\circ} \mathrm{C}$ for 3 days, and then sown onto half-strength Murashige and Skoog (MS) agar medium with $1 \%$ sucrose. Transformants were screened on $1 / 2 \mathrm{MS}$ agar medium containing $50 \mu \mathrm{g} / \mathrm{ml}$ Kanamycin. About two week old seedlings were transferred into soil. Plants were grown in phytotron under long-day conditions ( $8 \mathrm{~h} \mathrm{light} / 16 \mathrm{~h}$ dark) with light intensity $\left(100 \mu \mathrm{mol}\right.$ photons $\left.\mathrm{m}^{-2} \mathrm{~s}^{-1}\right)$ at $22^{\circ} \mathrm{C}$. 


\section{Plasmid construction and transformation}

The mutated SOT5 CDS (SOT5-m1 and SOT5-m2) was amplified from sot5 cDNA. The truncated EMB2654$11 M$ CDS (1-1635 bp) was amplified from WT cDNA. The truncated EMB976-7M CDS (1-1332 bp) and EMB976-14M CDS (1-2244 bp) were amplified from WT cDNA, respectively. In order to produce a truncated protein in the transgenic lines, we introduced a stop coden in the reverse primer when we amplifying $E M B 2654-11 M$, EMB976-7M and EMB976-14M sequences. The genomic PRPL4 (gPRPL4) was amplified from WT gDNA. The primer sequences were listed in Additional file 4. These amplified sequences were inserted into the pENTR $\mathrm{SD} / \mathrm{D}-\mathrm{TOPO}$ entry vector (Invitrogen). After sequencing, the corrected CDS fragments were recombined into the pGWB2 destination vector as previously described [8]. The destination vectors were transformed into Col-0 or rdr6-11 mutants using Agrobacterium strain GV3101 and the floral-dip method. Transformants were screened on $1 / 2 \mathrm{MS}$ agar medium containing $50 \mu \mathrm{g} / \mathrm{ml}$ Kanamycin.

\section{RNA isolation and RT-qPCR}

The chlorosis or albino leaves of each cosuppression lines were sampled for RNA extraction. Total RNAs were extracted from leaves of various lines according to the manufacturer's instructions (Promega Denaturing Solution, Z5651). Downstream DNAse I treatment was performed according to the manufacturer's instructions (Invitrogen/Gibco DNA-free DNase Treatment \& Removal Reagents, AM1906) and reverse transcription (RT) steps were conducted according to the manufacturer's instructions (Promega Reverse Transcription System, A3500). PCR was performed using the gene-specific primers that are listed in Additional file 4 and according to the manufacturer's instructions (TaKaRa Taq, R001WZ). Quantitative PCR was carried out using the gene-specific primers that are listed in Additional file 4 according to the manufacturer's instructions (Takara SYBR Premix Ex Taq, RR420; The Applied Biosystems MicroAmp ${ }^{\circledR}$ Fast Optical 96-Well Reaction Plate-0.1 ml, 4346906; ThermoFisher Scientific QuantStudio $^{\text {TM }} 3$ Real-Time PCR Systems). The data set was normalized using ACTIN2 as a reference. The method to quantify the transcript level and splicing efficiency of plastid genes was previously described $[8,30]$. The expression of endogenous EMB2279 genes was detected by the primer pair located in $5^{\prime} \mathrm{UTR}$ (Fig. 1). The total EMB2279 (including endogenous and transgenes) mRNA was detected by the primer pair located in the coding sequence. The expression of endogenous EMB2654 or EMB976 was detected by the primer pair located in the last half of its CDS (Fig. 1). The primers are listed in Additional file 4. In the figures of qPCR, the values are means of three technique replicates (bars indicate SD), asterisks indicate significant differences between wild type (WT) and transgenic plants (Student's $t$ test, ${ }^{*}, \mathrm{P}<0.05$ and $* *, \mathrm{P}<0.01$ ). At least two $\mathrm{T}_{1}$ transgenic lines were analyzed for each construct.

\section{Supplementary information}

Supplementary information accompanies this paper at https://doi. org/10.1186/s13007-020-00696-0.

Additional file 1: Figure S1. The gene is silenced specifically in each cosuppression line. a The expression level of EMB2654 in 35:SOT5-m 1/ Col-0 and 35:SOT5-m2/Col-0 cosuppression lines. b The expression level of EMB976 in 35:SOT5-m1/Col-0 and 35:SOT5-m2/Col-0 cosuppression lines. c The expression level of EMB2654 in 35S:EMB976-14M/Col-0 cosuppression lines. d The expression level of EMB976 in 35S:EMB2654-11M/Col-0 cosuppression lines.

Additional file 2: Figure S2. The phenotypes of transgenic plants overexpressing the alternative splicing variant (gPRPL4) CDS in WT background. a Chlorosis leaf phenotype of transgenic plants overexpressing gPRPL4 in WT background (bar $=2 \mathrm{~cm}$ ). $\mathbf{b}$ RT-PCR analysis of expression levels of the PRPL4 and PRPL28 in the typical cosuppression lines. The arrowhead indicates the transcripts of transgene $G P R P L 4$ and asterisk indicates the mature PRPL4 mRNA. The transgenic line 35S:CFM4/Col-0 is as a control. c RT-qPCR analysis of the mature PRPL4 mRNA in the typical cosuppression lines. $\mathbf{d}$ RT-qPCR analysis of expression levels of the PRPL28 in the typical cosuppression lines. It's noted that PRPL28 mRNA was not decreased in the 35S:gPRPL4/Col-0 cosuppression lines. For RT-qPCR, the values are means of three technique replicates (bars indicate SD). Asterisks indicate significant differences between wild type (WT) and transgenic plants (Student's t test, ${ }^{*}, \mathrm{P}<0.05$ and ${ }^{*}, \mathrm{P}<0.01$ ).

Additional file 3: Figure S3. The phenotypes of transgenic plants overexpressing the full length SOT5 CDS in the WT background. The chlorosis phenotype of the transgenic lines overexpressing the full length SOT5 CDS in the WT background appeared at the late developmental stage, such as the chlorosis inflorescence and cauline leaves. Overall, the chlorosis phenotype is not as serious as those cosuppression lines overexpressing the mutated SOT5 constructs.

Additional file 4. Primers used in this study.

\section{Abbreviations}

PPR: Pentatricopeptide-repeat proteins; EMB: Embryonic lethal; WT: Wild-type; PTGS: Post transcriptional gene silencing; RDR6: RNA-dependent RNA polymerase 6; DCL2: Dicer-like 2; DCL4: Dicer-like 4; RISC: RNA-induced silencing complex; RNAi: RNA interfering; CDS: Coding sequence; EMB2279: EMBRYO DEFECTIVE2279; SOT5: SUPPRESSOR OF THF1; EMB2654: EMBRYO DEFECTIVE2654; EMB976: EMBRYO DEFECTIVE976; SOT5-m1: SOT5 mutated protein 1; SOT5-m2: SOT5 mutated protein 2; EMB2654-11M: Truncated EMB2654 protein with 11 PPR motifs; EMB976-7M: Truncated EMB976 protein with 7 PPR motifs; EMB976-14M: Truncated EMB976 protein with 14 PPR motifs; RPL4: PLASTID RIBOSOMAL PROTEIN L4; PRPL28: PLASTID RIBOSOMAL PROTEIN L28; rpl2: Plastid ribosomal protein 12; rps 12: Plastid ribosomal protein s12; bp: Base pairs; nt: Nucleotide.

\section{Acknowledgements}

We thank Dr. Hongwei Guo for offering the rdr6-11 seeds and Dr. Qihua Ling for the helpful comments on the manuscript. 


\section{Authors' contributions}

WH conceived and designed the experiments; JC, $\mathrm{HZ}$ and $\mathrm{WH}$ performed the experiments; $\mathrm{WH}$ and $\mathrm{JH}$ supervised the experiments; JC, WH and $\mathrm{JH}$ wrote the paper. All authors read and approved the final manuscript.

\section{Funding}

This study was supported by grants from Shanghai Key Laboratory of Plant Molecular Sciences (18DZ2260500).

\section{Availability of data and materials}

All data generated or analyzed during this study are available in this published article and its additional files.

\section{Ethics approval and consent to participate}

Not applicable.

\section{Consent for publication}

Not applicable.

\section{Competing interests}

The authors declare that they have no competing interests.

Received: 18 June 2020 Accepted: 9 November 2020

Published online: 18 November 2020

\section{References}

1. Meinke DW. Genome-wide identification of EMBRYO-DEFECTIVE (EMB) genes required for growth and development in Arabidopsis. New Phytol. 2020;226(2):306-25.

2. Bryant N, Lloyd J, Sweeney C, Myouga F, Meinke D. Identification of nuclear genes encoding chloroplast-localized proteins required for embryo development in Arabidopsis. Plant Physiol. 2011;155(4):1678-89.

3. Lurin C, Andres C, Aubourg S, Bellaoui M, Bitton F, Bruyere C, Caboche M, Debast C, Gualberto J, Hoffmann B, et al. Genome-wide analysis of Arabidopsis pentatricopeptide repeat proteins reveals their essential role in organelle biogenesis. Plant Cell. 2004;16(8):2089-103.

4. Schmitz-Linneweber C, Small I. Pentatricopeptide repeat proteins: a socket set for organelle gene expression. Trends Plant Sci. 2008:13(12):663-70.

5. Sun YK, Gutmann B, Yap A, Kindgren P, Small I. Editing of chloroplast rps14 by PPR editing factor EMB2261 is essential for Arabidopsis development. Front Plant Sci. 2018:9:841

6. Lu Y, Li C, Wang H, Chen H, Berg H, Xia Y. AtPPR2, an Arabidopsis pentatricopeptide repeat protein, binds to plastid $23 \mathrm{~S}$ rRNA and plays an important role in the first mitotic division during gametogenesis and in cell proliferation during embryogenesis. Plant J. 2011;67(1):13-25.

7. Zhang J, Xiao J, Li Y, Su B, Xu H, Shan X, Song C, Xie J, Li R. PDM3, a pentatricopeptide repeat-containing protein, affects chloroplast development. J Exp Bot. 2017;68(20):5615-27.

8. Huang W, Zhu Y, Wu W, Li X, Zhang D, Yin P, Huang J. The pentatricopeptide repeat protein SOT5/EMB2279 is required for plastid rpl2 and trnK intron splicing. Plant Physiol. 2018;177(2):684-97.

9. Lee K, Park SJ, Colas des Francs-Small C, Whitby M, Small I, Kang H. The coordinated action of PPR4 and EMB2654 on each intron half mediates trans-splicing of rps 12 transcripts in plant chloroplasts. Plant J. 2019;100(6):1193-207.

10. Aryamanesh N, Ruwe H, Sanglard LV, Eshraghi L, Bussell JD, Howell KA, Small I, des Francs-Small CC. The pentatricopeptide repeat protein EMB2654 is essential for trans-splicing of a chloroplast small ribosomal subunit transcript. Plant Physiol. 2017;173(2):1164-76.

11. Wang X, Zhao L, Man Y, Li X, Wang L, Xiao J. PDM4, a pentatricopeptide repeat protein, affects chloroplast gene expression and chloroplast development in Arabidopsis thaliana. Front Plant Sci. 2020;11:1198.
12. Ossowski S, Schwab R, Weigel D. Gene silencing in plants using artificial microRNAs and other small RNAs. Plant J. 2008;53(4):674-90.

13. Schwab R, Ossowski S, Riester M, Warthmann N, Weigel D. Highly specific gene silencing by artificial microRNAs in Arabidopsis. Plant Cell. 2006;18(5):1121-33.

14. Liu W, Zhu X, Lei M, Xia Q, Botella JR, Zhu J-K, Mao Y. A detailed procedure for CRISPR/Cas9-mediated gene editing in Arabidopsis thaliana. Sci Bull. 2015;60(15):1332-47.

15. Mao Y, Zhang H, Xu N, Zhang B, Gou F, Zhu JK. Application of the CRISPR-Cas system for efficient genome engineering in plants. Mol Plant. 2013:6(6):2008-11.

16. Schubert D, Lechtenberg B, Forsbach A, Gils M, Bahadur S, Schmidt R. Silencing in Arabidopsis T-DNA transformants: the predominant role of a gene-specific RNA sensing mechanism versus position effects. Plant Cell. 2004;16(10):2561-72

17. van der Krol AR, Mur LA, Beld M, Mol JN, Stuitje AR. Flavonoid genes in petunia: addition of a limited number of gene copies may lead to a suppression of gene expression. Plant Cell. 1990;2(4):291-9.

18. Napoli C, Lemieux C, Jorgensen R. Introduction of a chimeric chalcone synthase gene into petunia results in reversible co-suppression of homologous genes in trans. Plant Cell. 1990;2(4):279-89.

19. Borges F, Martienssen RA. The expanding world of small RNAs in plants. Nat Rev Mol Cell Biol. 2015;16(12):727-41.

20. Bologna NG, Voinnet O. The diversity, biogenesis, and activities of endogenous silencing small RNAs in Arabidopsis. Annu Rev Plant Biol. 2014:65:473-503.

21. Zhang C, Zhang W, Ren G, Li D, Cahoon RE, Chen M, Zhou Y, Yu B, Cahoon EB. Chlorophyll synthase under epigenetic surveillance is critical for vitamin E synthesis, and altered expression affects tocopherol levels in Arabidopsis. Plant Physiol. 2015;168(4):1503-11.

22. Peragine A, Yoshikawa M, Wu G, Albrecht HL, Poethig RS. SGS3 and SGS2/ SDE1/RDR6 are required for juvenile development and the production of trans-acting siRNAs in Arabidopsis. Genes Dev. 2004;18(19):2368-79.

23. Mallory AC, Vaucheret $H$. ARGONAUTE 1 homeostasis invokes the coordinate action of the microRNA and siRNA pathways. EMBO Rep. 2009;10(5):521-6.

24. Que Q, Wang HY, English JJ, Jorgensen RA. The frequency and degree of cosuppression by sense chalcone synthase transgenes are dependent on transgene promoter strength and are reduced by premature nonsense codons in the transgene coding sequence. Plant Cell. 1997;9(8):1357-68.

25. Chang YF, Imam JS, Wilkinson MF. The nonsense-mediated decay RNA surveillance pathway. Annu Rev Biochem. 2007;76:51-74.

26. Hogg JR, Goff SP. Upf1 senses 3'UTR length to potentiate mRNA decay. Cell. 2010;143(3):379-89.

27. Luo Z, Chen Z. Improperly terminated, unpolyadenylated mRNA of sense transgenes is targeted by RDR6-mediated RNA silencing in Arabidopsisle. Plant Cell. 2007;19(3):943-58.

28. Sakamoto W. Protein degradation machineries in plastids. Annu Rev Plant Biol. 2006;57:599-621.

29. Kuroda H, Maliga P. The plastid clpP1 protease gene is essential for plant development. Nature. 2003;425(6953):86-9.

30. de Longevialle AF, Hendrickson L, Taylor NL, Delannoy E, Lurin C, Badger M, Millar AH, Small I. The pentatricopeptide repeat gene OTP51 with two LAGLIDADG motifs is required for the cis-splicing of plastid ycf3 intron 2 in Arabidopsis thaliana. Plant J. 2008;56(1):157-68.

31. Wu W, ZhuY, Ma Z, Sun Y, Quan Q, Li P, Hu P, Shi T, Lo C, Chu IK, et al. Proteomic evidence for genetic epistasis: ClpR4 mutations switch leaf variegation to virescence in Arabidopsis. Plant J. 2013;76(6):943-56.

\section{Publisher's Note}

Springer Nature remains neutral with regard to jurisdictional claims in published maps and institutional affiliations. 